\title{
Effects of Finite Deformed Length in Carbon Nanotubes
}

\author{
Jun-Qiang Lu, ${ }^{1}$ Jian Wu, ${ }^{1}$ Wenhui Duan, ${ }^{1}$ and Bing-Lin $\mathrm{Gu}^{1}$ \\ ${ }^{1}$ Center for Advanced Study and Department of Physics, Tsinghua University, Beijing 100084, China
}

(Dated: November 16, 2018)

\begin{abstract}
The effect of finite deformed length is demonstrated by squashing an armchair $(10,10)$ singlewalled carbon nanotube with two finite tips. Only when the deformed length is long enough, an effectual metal-semiconductor-metal heterojunction can be formed in the metallic tube. The effect of finite deformed length is explained by the quantum tunnelling effect. Furthermore, some conceptual designs of nanoscale devices are proposed from the metal-semiconductor-metal heterojunction.
\end{abstract}

The discovery of carbon nanotubes[1] offers a natural quasi one dimensional material to explore and verify quantum physics theory, and the advantage in design of nanodevice. Recently, an interesting topic is how a mechanical deformation of a single-walled carbon nanotube (SWNT) affects its electronic property. 2, 3, 4, 5, 5] 6] Some previous studies [5, 6] show that in many cases, a local deformation can not drive a metal-to-semiconductor transition (MST) in armchair SWNTs. At the same time, our recent work 7] indicates that a MST can be achieved by a radial deformation along the holistic armchair SWNTs. The different conclusions drawn from the two models show that the finiteness of the deformed length plays an important role in the transport properties of SWNTs. So, for designing electronic devices based on nanotubes, the deformed length along the tube will be a key parameter to ensure their functions. Here, in this letter, we will demonstrate the effects of the deformed length by squahing an armchair $(10,10)$ SWNT.

In order to simplify the theoretical analysis, we consider an armchair $(10,10)$ SWNT symmetrically squashed by two identical tips (with a width of $d_{x}=4.0 \AA$ and a length of $d_{z}$ ) along the $\pm y$ direction till $d_{y}=2.2 \AA,[7]$ as shown in Figs. 1a and 1b. The atomic structures, or the shape of tubes, for different tip lengths $d_{z}$, are optimized by the tight-binding (TB) molecular dynamics (MD) code. 7, 8] Fig. 1a shows an example optimized structure for tip length $d_{z}=6.0 \AA$. Noticeably, the cross section of the tube changes from a dumbbell (Fig. 1b, corresponding to fully deformed part of the tube) to an ellipse (Fig. 1c, less deformed part), and then to a circle (Fig. 1d, undeformed part). The smallest interatomic distance between the upper face and the lower face is $d_{A A^{\prime}}=2.1 \AA$, and the mirror symmetry is broken due to small $d_{y}$. In this case, the squashed part should work as semiconductors. [7]

After obtaining the optimized structure with different tip lengths, we employ the TB Green's function method [9, 10, 11] to study the electronic transport properties of the deformed tubes. Fig. 2a presents the conductance curves near the Fermi energy $E_{F}$ of tubes deformed by tips with different lengths $d_{z}$. We can see that no energy gap is opened when $d_{z}$ is $3.0 \mathrm{~nm}$ (the pink short dash line). In other words, the deformed tube keeps its metallic behavior. Moreover, the conductance of the deformed tube almost keeps constant near $E_{F}$. Only when the tip length increases, the conductance near $E_{F}$ will begin to decrease, as shown in Fig. 2a. It is easy to find that the conductance near $E_{F}$ approaches zero when $d_{z}=25.2 \mathrm{~nm}$ (the red dash line). As the limit, when the length of tips approaches infinite, the whole tube is deformed, and then the conductance near $E_{F}$ will be zero.

The above results demonstrate that the deformed length in SWNTs plays an important role in their electronic properties. In order to reveal the effects of finite deformed length in SWNTs, we present a visual picture by the local density of states (LDOS) 10] per unit cell layer along the tube axes. Each unit cell layer consists of 40 atoms which distribute over two atomic layers. When $d_{z}=25.2 \mathrm{~nm}$, the LDOS of 120 unit cell layers (4800 atoms) is plotted in Fig. 2b, as the deformed part of the tube contains about 100 unit cell layers. It is obvious that the LDOS of the deformed part is almost zero near $E_{F}$. Energy gap exists near $E_{F}$ within the deformed part. While, the LDOS crosses $E_{F}$ continuously in the nearby part. Consequently, the squashed tube forms a metal-semiconductor-metal (MSM) heterojunction. Meanwhile, when $d_{z}=3.0 \mathrm{~nm}$, the LDOS of 30 unit cell layers (1200 atoms) is plotted in Fig. 2c, since the deformed part only includes about 12 unit cell

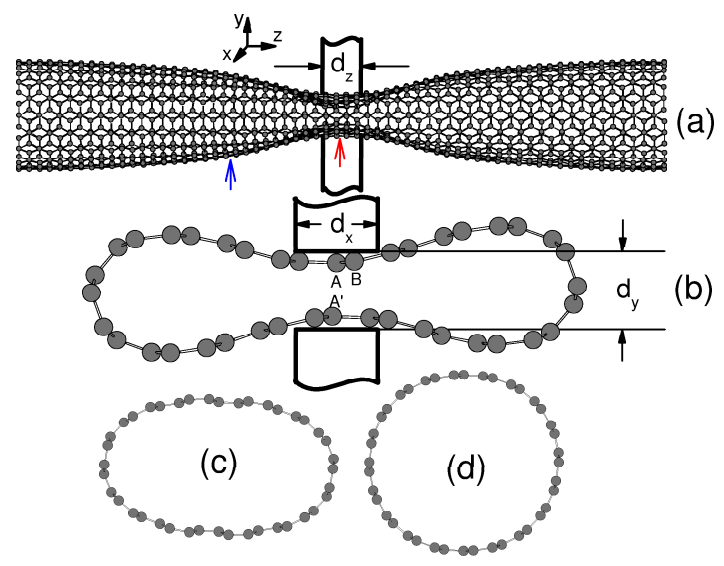

FIG. 1: (color) (a) Finite length deformation in an armchair $(10,10)$ SWNT squashed by two identical tips; (b)(c) Cross sections of the different parts of the deformed SWNT, as indicated by the red and blue arrows, respectively; (d) Cross section of the non-deformed part of the SWNT. 

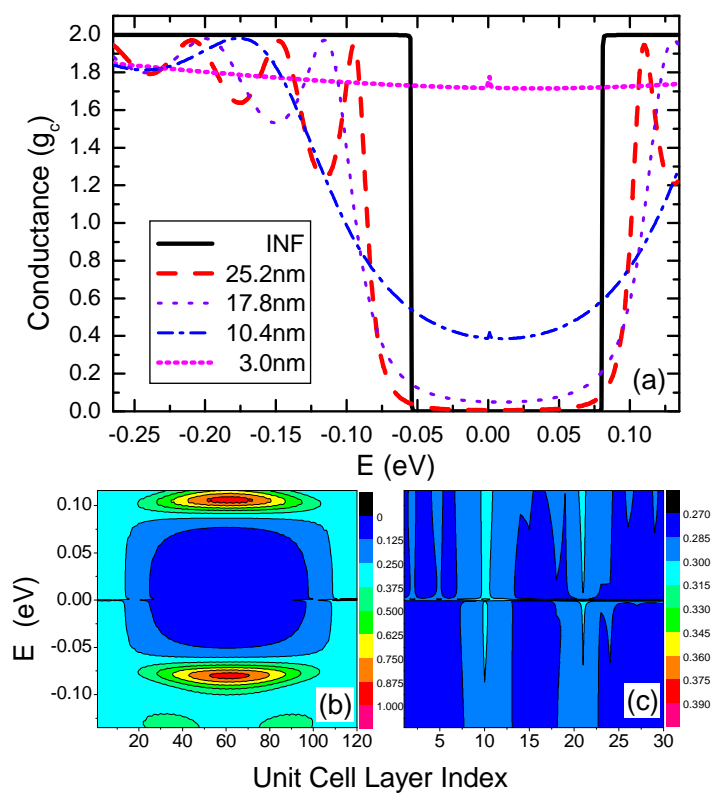

FIG. 2: (color) (a) Conductances of SWNTs deformed by tips with different length $d_{z} \cdot g_{c}\left(=2 e^{2} / h\right)$ is the unit quanta of conductance. $E$ is the energy of injected electron, and the Fermi energy of ideal armchair $(10,10)$ SWNT is taken as zero. (b)(c) The LDOS (unit: $\mathrm{eV}^{-1}$ ) per unit cell layer near $E_{F}$ of SWNTs deformed by tips with lengths (b) $d_{z}=25.2 \mathrm{~nm}$ and (c) $d_{z}=3.0 \mathrm{~nm}$.

layers. The LDOS crosses $E_{F}$ continuously in both the deformed part and its nearby. Hence, the tube keeps its metallic behavior.

Physically, the effect of finite deformed length can be understood by the quantum tunnelling effect. It is well known that the tunnelling probability attenuate exponentially with increasing width of energy barrier. Obviously, electrons can easily tunnel through the energy barrier if it is narrow enough. This also occurs for nanoelectronic devices. If the size of the device is too small, the electron will tunnel through the device, and consequently, the device can not function well as expected. The size effect is an essential problem for nanoelectronic devices design, as the tunnelling effect is universal.

To give a vivid sight of the tunnelling effect in the deformed tube, we present the LDOS of each atom within one unit cell layer of the dumbbell part in Figs. 3a and $3 \mathrm{~b}$. It is well known that the graphene sheet and hence the nanotube have two equivalent sublattices, which may be labelled as A and B sublattices. It has been pointed out that, when a MST is achieved in a squashed armchair SWNT, the LDOS distributing on the two sublattices are distinguishable, resulting in a discontinuity energy spectrum near $E_{F}$. 7] This phenomena can also be found in the dumbbell part of tube deformed by tips with length $d_{z}=25.2 \mathrm{~nm}$, as shown in Fig. 3a. For the case of $d_{z}=3.0 \mathrm{~nm}$, the LDOS distribution of the dumbbell part is presented in Fig. 3b. We can find only a slight dis- tinction between the LDOS of the two sublattices. While the LDOS crosses $E_{F}$ continuously. The LDOS near $E_{F}$ in the dumbbell part should result from the tunnelling effect.

More interesting thing is the change of electronic structure of the tube due to the finite deformation. The LDOS within a unit cell layer of the ellipse part (as shown in Fig. 1c) of the tube deformed by tips with $d_{z}=25.2$ $\mathrm{nm}$ is plotted in Fig. 3c. Though there is no energy gap near $E_{F}$ with the absence of the bond formation between the flattened faces, it is still interesting to find that the LDOS over the two sublattices are also distinguishable, which results from the distinction of the two sublattices in dumbbell part. To reveal this effect, in Fig. 3d, we plot the LDOS within a circle unit cell layer, which is far from the deformed center with distance $40.6 \mathrm{~nm}$. To our surprise, again, the LDOS of the two sublattices are distinguishable. And more strange thing is the LDOS on one certain atom oscillates with energy, as indicated by the red dash line in Fig. 3d. Further studies show that the LDOS of the two sublattices within a unit cell layer are always distinguishable when the distance between the layer and the deformed center increases, and the oscillation of LDOS with energy becomes more exquisite. For an ideal SWNT, the LDOS near $E_{F}$ distribute over each carbon atom homogeneously. With the finite deformation, the $\pi$ and $\pi^{*}$ wave functions in the tube are mixed due to the breaking of the mirror symmetry. [7] At the same time, as the translation symmetry along the tube is also broken, the standing wave functions are the combination of the incoming and reflecting wave functions, [1] and the LDOS of them can be expressed as $2[1 \pm \cos (2 \Delta k x)]\left[1+R \pm 2 \sqrt{R} \cos \left(2 k_{F}^{\prime} x+\delta\right)\right] .[12]$ Obviously, the distinction between the LDOS of the two sub-

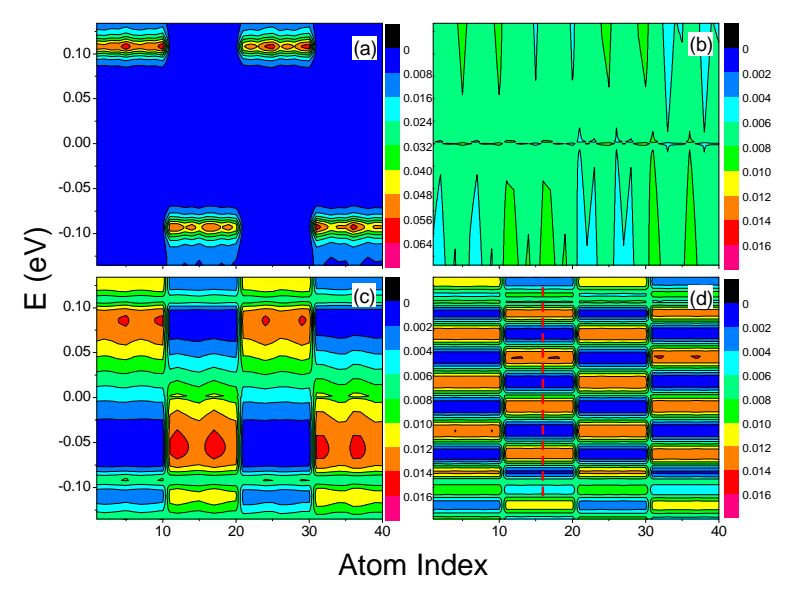

FIG. 3: (color) The LDOS (unit: $\mathrm{eV}^{-1}$ ) near $E_{F}$ within a unit cell layer of SWNTs deformed by tips with different lengths (a)(c)(d) $d_{z}=25.2 \mathrm{~nm}$ and (b) $d_{z}=3.0 \mathrm{~nm}$. (a)(b) dumbbell part; (c) ellipse part and (d) circle part. The atoms in B (A) sublattice are labelled 1 (11) through 10 (20) for the first atomic layer and 21 (31) through 30 (40) for the second atomic layer. The dash line is added only for guide. 
lattices results from the difference between $1 \pm \cos (2 \Delta k x)$. For a certain $x$, the oscillation of LDOS with energy results from $\cos (2 \Delta k x)$. As the energy dispersion relations near $E_{F}$ is approximately linear with $\Delta k=2 E /\left(\sqrt{3} a \gamma_{0}\right)$, the period of the oscillation is $T_{E}=\sqrt{3} a \gamma_{0} \pi /(2 x)$.

From the above discussion, we know that an armchair $(10,10)$ SWNT can form a MSM heterojunction when the deformation length is large enough $\left(d_{z} \sim 20 \mathrm{~nm}\right)$. For the conducting electrons, the middle semiconductor layers act as a energy barrier. Thus, based on the MSM heterojunction, we can easily structure some SWNT-based nanoscale devices.

Double barrier - To structure nanoscale double barrier, we only need to deform an armchair SWNT at two different positions, as shown schematically in Fig. 4a. As well known, the typical behavior of double barrier structure is the resonant tunnelling. We find that the conductance exhibits such a behavior (see Fig. 4a). The small conductance peaks lying in the energy gap are due to the resonant tunnelling effect. And, it is easy to control the electronic transport property of the nanoscale double barrier through changing the widths of the two energy barriers and the middle energy trap (i.e., changing the deformed length and the distance between the two deformed positions). For the example case shown in Fig. 4a , the tip length $d_{z}=25.2 \mathrm{~nm}$, and the distance between the two deformed positions $l=57.1 \mathrm{~nm}$.

Superlattice - If an armchair SWNT is deformed periodically along its axis, as shown schematically in Fig. $4 \mathrm{~b}$, it will behave as a superlattice. The conductance of the nanoscale superlattice is also presented in Fig. $4 \mathrm{~b}$. The period of the superlattice is $l=57.1 \mathrm{~nm}$, and the tip length is $d_{z}=25.2 \mathrm{~nm}$. Each conductance peak is split into many small peaks, as shown in the inset. This is the feature of superlattice structure, named as resonant splitting effect. It is also found that the conductance vanishes near $E_{F}$ between the conductance bands. We can control the electronic transport through changing the range of the conductance gap, which can be achieved by adjusting the structure parameters.

Transistor - For a logical circuit, transistor is one of the most important components. Designing a nanoscale transistor will be one of key steps to realize the nanologic. Recently, Bachtold et al. achieved a nanoscale transistor experimentally. 13. They adopted a semiconducting SWNT. The SWNT needs to be connected with two gold electrodes. Here, based on the MSM heterojunction, we can design pure carbon nanoscale transistor.

Fig. $4 \mathrm{c}$ is the schematics of a conceptual design of nanoscale transistor $\left(d_{z}=25.2 \mathrm{~nm}\right)$. If a gate voltage $V_{g}$ is applied to the tips, the MSM heterojunction will act as a nanoscale transistor. When $V_{g}=0$, as the deformed part behaves as semiconductor, the conductance is zero and the transistor is OFF. With the change of $V_{g}$, the energy gap of the deformed part shifts. When $E_{F}$ does not lie in the energy gap any longer, the transistor begins to be ON. That is the basic principle of our nanoscale transistor. The dependence of the conductance with $V_{g}$
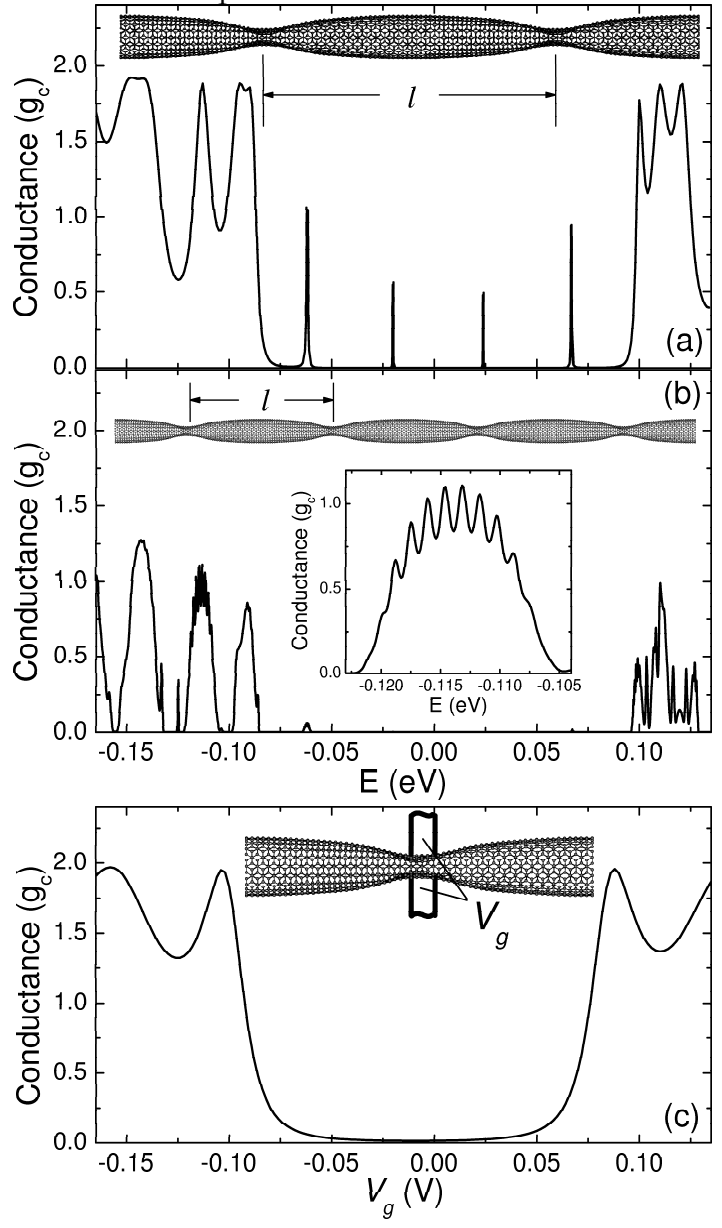

FIG. 4: Conceptual designs of nanoscale (a) double barrier; (b) superlattice; (c) transistor and their conductance curves. Note that the structures are shown schematically only. The inset presents the detail of resonant splitting effect.

is also presented in Fig. 4c. We can find the logical voltage of the transistor is about $-0.10 \mathrm{~V}$.

In summary, we demonstrate the effect of finite deformed length through squashing an armchair $(10,10)$ SWNT. Only a deformation with enough long length can lead to an effectual MSM heterojunction in the metallic tube. The effect of finite deformed length is a general problem and is explained by the quantum tunneling effect. The change of the electronic structure, or the LDOS distributions, due to the finite deformation, shows the correlation between the nanodevices and leads. It should be considered in design of nanologic. Furthermore, based on the MSM heterojunction, some conceptual designs of nanoscale devices are proposed.

The work is supported by the National High Technology Research and Development Program of China (Grant No. 2002AA311153), the Ministry of Education of China, and the National Natural Science Foundation of China. 
[1] S. Iijima, Nature (London) 354, 56 (1991).

[2] T.W. Tombler, C. Zhou, L. Alexseyev, J. Kong, H. Dai, L. Liu, C.S. Jayanthi, M. Tang, and S. Wu, Nature (London) 405, 769 (2000).

[3] E.D. Minot, Y. Yaish, V. Sazonova, J.Y. Park, M. Brink, and P.L. McEuen, Phys. Rev. Lett. 90, 156401 (2003).

[4] J. Cao, Q. Wang, and H. Dai, Phys. Rev. Lett. 90, 157601 (2003).

[5] A. Maiti, A. Svizhenko, and M.P. Anantram, Phys. Rev. Lett. 88, 126805 (2002).

[6] M.B. Nardelli and J. Bernhole, Phys. Rev. B 60, R16338 (1999).

[7] J.Q. Lu, J. Wu, W. Duan, F. Liu, B.F. Zhu, and B.L. Gu, Phys. Rev. Lett. 90, 156601 (2003).
[8] C.H. Xu, C.Z. Wang, C.T. Chan, and K.M. Ho, J.Phys.: Condens. Matter 4, 6047 (1992).

[9] J.C. Charlier, Ph. Lambin, and T.W. Ebbesen, Phys. Rev. B 54, R8377 (1997).

[10] J. Wu, B.L. Gu, H. Chen, W. Duan, and Y. Kawazoe, Phys. Rev. Lett. 80, 1952 (1998).

[11] J. Wu, W. Duan, B.L. Gu, J.Z. Yu, and Y. Kawazoe, Appl. Phys. Lett. 77, 2554 (2000).

[12] H.F. Song, J.L. Zhu, and J.J. Xiong, Phys. Rev. B 66, 245421 (2002).

[13] A. Bachtold, P. Hadley, T. Nakanishi, and C. Dekker, Science 294, 1317 (2001). 